\title{
FORMAÇÃO DO PROFESSOR DA EDUCAÇÃO BÁSICA NA PERSPECTIVA DA APRENDIZAGEM CRIATIVA
}

\author{
Elisabete Aparecida Alves SOARES ${ }^{\mathrm{i}}$ \\ Maria Elisabette Brisola Brito PRADOii \\ Fátima Aparecida da Silva DIAS ${ }^{i i i}$
}

\begin{abstract}
RESUMO
Este trabalho objetiva analisar o impacto das ações da Diretoria de Políticas e Tecnologias Educacionais da Secretaria de Estado de Educação do Paraná, entre 2016 e 2018, à luz do conceito Aprendizagem Criativa e da teoria dos 4 Ps. Também se pauta no modelo teórico Technological Pedagogical Content Knowledge (TPACK), que sistematiza a intersecção entre conhecimentos pedagógicos, de conteúdo e tecnológicos necessários a uma ação docente integrada à tecnologia. A metodologia qualitativa envolveu a descrição de um processo formativo de professores do estado do Paraná. Os dados foram coletados ao término do curso. A observação da formação continuada dos professores, abordando metodologias ativas, demonstrou dificuldade em aproximar tais metodologias dos conteúdos curriculares, assim como a complexidade de se implementar práticas inovadoras numa cultura escolar alicerçada por práticas tradicionais.
\end{abstract}

PALAVRAS-CHAVE: Formação Continuada; Aprendizagem Criativa; TPACK; Currículo.

\section{TEACHER TRAINING OF BASIC EDUCATION FROM THE PERSPECTIVE OF CREATIVE LEARNING}

\begin{abstract}
This paper aims to analyze the impact of the actions of the Directory of Policies and Educational Technologies of the State Department of Education of Paraná, between the years 2016 to 2018, in the light of the concept of Creative Learning and the theory of the 4 Ps. It is also based on the theoretical model Technological Content Pedagogical Knowledge (TPACK), which summarises the intersection among pedagogical, content and technological knowledge necessary for a teaching action integrated with technology. The methodology of this qualitative study involved the description of a formative process of teachers from the state of Paraná. Data were collected after finishing the course. The observation of the teachers continuous training addressing active methodologies demonstrated the difficulty in bringing such methodologies closer to the curriculum contents, as well as the complexity of implementing innovative practices in a school culture based on traditional practices.
\end{abstract}

KEYWORDS: Continuing Education; Creative Learning; TPACK; Resume.

\footnotetext{
${ }^{\text {i }}$ Doutoranda em Metodologias para o Ensino de Linguagens e suas Tecnologias da UNOPAR - PR. E-mail: elisa1210@gmail.com.

ii Doutorado em Educação: Currículo. Docente no Programa de Pós-Graduação em Educação Matemática da Universidade Anhanguera de São Paulo e do Programa de Pós-Graduação em Metodologias para o Ensino de Linguagens e suas Tecnologias da UNOPAR - PR. E-mail: bette.prado@gmail.com.

iii Doutorado em Educação Matemática. Docente do Programa de Pós-Graduação em Metodologias para o Ensino de Linguagens e suas Tecnologias da UNOPAR - PR. E-mail: fatima.a.dias@kroton.com.br.
} 


\section{FORMACIÓN DEL PROFESORADO /DE EDUCACIÓN BÁSICA DESDE LA PERSPECTIVA DEL APRENDIZAJE CREATIVO}

\section{RESUMEN}

El trabajo tiene como objetivo analizar el impacto de las acciones de la Dirección de Políticas y Tecnologías Educativas del Departamento de Educación del Estado de Paraná, entre los años 2016 a 2018, a la luz del concepto Deaprendizaje Creativo y la teoría de las 4 Ps. También se basa en el modelo teórico Technological Content Pedagogical Knowledge (TPACK), que sistematiza la intersección entre los conocimientos pedagógicos, contenidos y tecnológicos necesarios para una acción docente integrada con la tecnología. La metodología de este estudio cualitativo implicó la descripción de un proceso formativo de profesores del estado de Paraná. Los datos se recopilaron al final del curso. La observación de la educación continua de los docentes, que abordan metodologías activas, demostró la dificultad de acercar dichas metodologías a los contenidos curriculares, así como la complejidad de implementar prácticas innovadoras en una cultura escolar basadas en las prácticas tradicionales.

PALABRAS CLAVE: Educación Continua; Aprendizaje Creativo; TPACK; Reanudar.

\section{INTRODUÇÃO}

Parece ser consenso que vivemos a era das tecnologias digitais, da computação, da sociedade em rede, e há tempos prega-se a intensa influência que toda essa evolução tecnológica tem exercido sobre a humanidade.

Nesse cenário, inúmeras teorias sobre novas formas de aprender e de ensinar originam metodologias diversificadas e suas ramificações, tais como: Aprendizagem Criativa, Movimento Maker, Ensino Híbrido, Linguagem de programação, Gamificação, entre outras.

A Aprendizagem Criativa busca apoiar-se na ressignificação das estratégias da educação infantil em qualquer nível de ensino, propondo atividades lúdicas e trabalho por pares com o objetivo de que os alunos aprendam de forma prazerosa e baseados nos seus interesses por meio de desafios de construção e raciocínio, utilizando materiais de diversas naturezas.

Essa forma de aprender se constitui como um processo de construção e reconstrução do conhecimento, que pode ser desenvolvido pela vivência dos alunos em atividades voltadas à resolução colaborativa de problemas, instigando a experimentação e o desafio para criação de novas soluções tendo como foco a interdisciplinaridade.

Nesse contexto, o papel do professor passa a ser o de orientador, mediador, articulador, provocador, inspirador, colaborador, pesquisador enquanto o aluno se torna o protagonista, criador, explorador, questionador, produtor, aprende por meio da prática e do erro. Alicerçada 
pelo Construcionismo, uma teoria proposta por Seymour Papert (1986), matemático, criador da Metodologia Logo - que utiliza uma linguagem de programação voltada para crianças e adultos das diferentes áreas de conhecimento, além de ser um dos pioneiros da Inteligência Artificial.

Papert trabalhou com Piaget na Universidade de Genebra de 1958 a 1963 e observando o comportamento de um grupo de alunos durante uma aula de Arte, Papert começou a delinear uma abordagem de educação, o Construcionismo, pois desejava que as crianças tivessem a mesma postura também nas aulas de Matemática, visto que nos projetos daquela disciplina havia engajamento, os alunos podiam pensar, contemplar, experimentar, tentar várias ideias, compartilhar os trabalhos com os colegas, enfim, ele queria que os estudantes pudessem se envolver de maneira semelhante com a Matemática (PAPERT; HAREL, 1991).

No Construcionismo há especial valorização do que se pode externar através do processo de elaboração mental, desta forma, não é uma teoria de caráter mentalista ou intelectual apenas, mas sim uma proposta que reúne o trabalho de idealização do projeto e sua materialização por meio dos recursos disponíveis. De acordo com Papert e Harel (1991), “o Construcionismo, de uma forma sucinta, poderia ser caracterizado como um modo de aprendizado que pede a construção de algo para que se possa compreender o seu funcionamento" (SCHELLER, VIALI, LAHM, 2014, p. 5).

Acreditando na atividade de o aluno ensinar algo para a máquina executar - programar o computador - Papert inaugurou no final da década de 60 uma nova abordagem educacional fazendo uso da linguagem Logo. Para programar, o aluno (de diferentes níveis de escolaridade) descreve os procedimentos que deseja ver executado pela máquina, usando os comandos da linguagem computacional Logo e, imediatamente, pode constatar o resultado da sua descrição sendo executada na tela do computador. Diante desse resultado, o aluno é instigado a rever e a refletir sobre o próprio pensamento expresso na descrição - a qual envolve estratégias e conceitos. A reflexão que ocorre nesse momento é fundamental para que ele possa vivenciar o processo de depuração sobre suas ideias, podendo buscar novas compreensões.

Valente (2016) destaca, com base nas ideias de Papert, que "os computadores deveriam ser utilizados para que as pessoas pudessem "pensar com" as máquinas e "pensar sobre" o próprio pensar (p.869). Isto significa que a programação Logo, desenvolvida na abordagem 
Construcionista, permite ao aluno a aprender com a mão na massa e a mente envolvida, ou seja, por meio do hands-on e head-in, como destaca Ackermann (1993).

\section{A TEORIA DOS 4 PS - PROJETOS, PARCERIAS, PAIXÃO, PENSAR BRINCANDO}

Mitchel Resnick ${ }^{1}$, pesquisador do MIT (Massachusetts Institute of Technology) e seguidor da teoria do construcionismo de Papert, defende que aliar a criatividade e a aprendizagem, como geralmente acontece na Educação Infantil, deveria ser o caminho percorrido também no Ensino Fundamental e Médio na busca do conhecimento. Resnick defende a lógica do "Lifelong Kindergarten" (Jardim da infância para toda a vida) integrando atividades de pesquisa e criação às disciplinas do currículo, levando os alunos a descobrirem soluções para os problemas do dia a dia e tornando a aprendizagem mais significativa para eles. Inspirado por essa visão, Resnick (2014) amplia as ideias construcionistas de Papert e propõe uma abordagem educacional, voltada para a constituição de um ambiente de aprendizagem com quatro pilares denominados de 4 Ps: Projects, Peers, Passion, and Play (Projetos, Parcerias, Paixão, Pensar brincando):

Projetos: as pessoas aprendem melhor quando estão trabalhando ativamente em projetos que sejam significativos para elas - gerando novas ideias, criando protótipos e aperfeiçoando-os sempre que necessário.

Parcerias: O aprendizado é mais frutífero quando inserido num contexto social, onde as pessoas compartilham ideias, colaboram em projetos e cada um constrói a partir do trabalho do outro.

Paixão: quando as pessoas participam de projetos com temas do seu interesse, que são realmente importantes para si, elas trabalham com mais dedicação e persistência diante dos desafios.

Pensar brincando: aprender envolve experimentação livre - buscar coisas novas, explorar materiais, testar fronteiras, correr riscos e fazer muitas vezes de diferentes formas.

Essas características da aprendizagem criativa requerem a criação de novos espaços e reorganização das atividades escolares a fim de promover um aprendizado significativo baseado em projetos, instigando os estudantes a gerenciar o seu próprio aprendizado. Nessa abordagem, é necessário oportunizar aos estudantes vivenciar situações em que possam descobrir, 
transformar, representar, imaginar, construir, praticar, testar, errar, refazer, compartilhar, observar, experimentar, reformular, redescobrir, ou seja, aprender a aprender de forma contínua.

Assim, considerando que essa nova forma de aprender demanda a formação continuada do professor para reconstruir a sua prática, este artigo tem como objetivo analisar o impacto das ações da Diretoria de Políticas e Tecnologias Educacionais (DITEC) da Secretaria de Estado de Educação do Paraná (SEED), entre os anos 2016 e 2018, à luz do conceito Aprendizagem Criativa e da teoria dos 4 Ps desenvolvida pelo professor Mitchel Resnick do MIT. O presente estudo também se pauta no modelo teórico TPACK (Technological Pedagogical Content Knowledge) ou Conhecimento Tecnológico e Pedagógico do Conteúdo criado pelos pesquisadores Mishra e Koehler (2006), o qual estabelece uma abordagem metodológica baseada na intersecção entre os conhecimentos pedagógicos, de conteúdo e tecnológicos.

\section{FORMAÇÃO E O CONHECIMENTO PROFISSIONAL DO PROFESSOR}

Aliado à necessidade de formação dos profissionais que atuam na ponta com a educação básica, vê-se a crescente discussão que tem tomado os setores acadêmicos acerca de teorias capazes de preencher lacunas que inviabilizam a efetiva transposição didática de metodologias com o uso de tecnologias na sala de aula.

Muitas são as vertentes que se debruçam sobre a temática e procuram dar visibilidade a propostas educacionais subsidiadas pelo uso de tecnologias de forma pedagógica ou metodologias de ensino classificadas como ativas, contemporâneas, criativas, híbridas, enfim, alternativas para a esfera educacional que pretendem alterar a dinâmica da sala de aula, fugindo das pedagogias essencialmente tradicionais e conferindo protagonismo ao aluno, até então, tido como mero espectador, receptor de conteúdos.

Neste contexto de pesquisas, emerge um modelo teórico representativo do conhecimento profissional do professor que deve e pode ser norteador das ações formativas voltadas para o uso das tecnologias no contexto educacional. Este modelo teórico denominado TPACK (Technological Pedagogical Content Knowledge) ou Conhecimento Tecnológico e 
Pedagógico do Conteúdo criado pelos pesquisadores Mishra e Koehler (2006), sistematiza a intersecção entre os conhecimentos pedagógicos, de conteúdo e tecnológicos necessários a uma ação docente integrada à tecnologia.

O TPACK caracteriza-se como um postulado científico que reúne os elementos essenciais para que a prática docente com o uso das tecnologias se concretize de forma integrada aos conteúdos curriculares. Baseados nos estudos de Shulman (1987), mais especificamente no Conhecimento Pedagógico do Conteúdo, Mishra e Koehler (2006) basicamente acrescentaram o conhecimento tecnológico, propondo a intersecção da tríade: conhecimento pedagógico, conhecimento do conteúdo e conhecimento tecnológico, sinalizando para a necessidade da apropriação tecnológica por meio da visão abrangente entre os saberes didáticos, metodológicos, cognitivos e a estrutura educacional vigente (pedagógicos), os saberes específicos curriculares a serem ensinados (de conteúdo) e os saberes técnico-pedagógicos acerca das tecnologias mais adequadas aos objetivos de aprendizagem (tecnológicos). A figura 1, a seguir, ilustra as interseções dos conhecimentos constituintes do TPACK. 


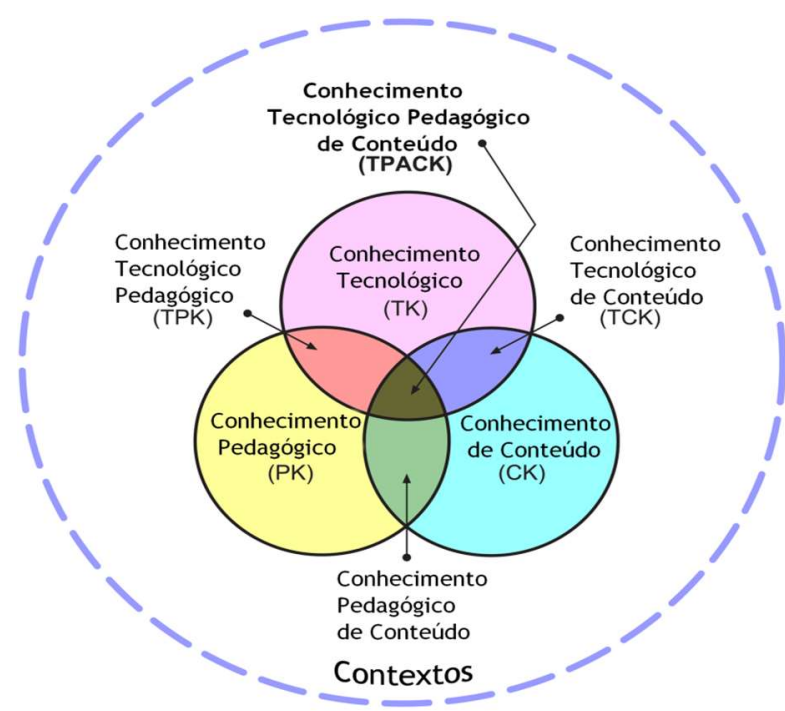

Figura 1 - TPACK - Technological Pedagogical Content Knowledge Fonte: Koehler, M.J.; Mishra (2009, p. 63).

Balizados por esta linha teórica, o TPACK, é possível compreender que práticas pedagógicas com pretensões inovadoras e uso de tecnologias não recaem apenas no uso técnicopedagógico de um determinado recurso, para além disso, estabelecem relação de interdependência com conhecimentos relacionados aos modos de aprender dos indivíduos, fortemente influenciados por questões sócio-histórico-ideológicas, e aos sistemas de ensino enquanto organizações estruturais do estado, aliados ao domínio do objeto de conhecimento a ser sistematizado. A partir do TPACK, é possível traçar o perfil desejável do uso de tecnologias no ambiente escolar como uma prática não isolada de outras instâncias educacionais (pedagógicas e de conteúdo) e deslocada do contexto de ensino e aprendizagem regular como alternativas aos imprevistos corriqueiros de uma escola (aulas vagas, projetos temáticos ou de contraturno etc), sem o devido planejamento e com fim em si mesmo.

No Brasil, pode-se dizer que as primeiras iniciativas em termos de inserção da tecnologia no meio educacional aconteceram timidamente nos anos 80 e a sua disseminação como política pública do governo federal se deu principalmente por meio dos programas PRONINFE 1987 e PROINFO 1997², portanto, são relativamente atuais. Nesse ínterim, os profissionais, na maioria das vezes, ainda se formam nas licenciaturas discutindo pouco ou quase nada sobre metodologias inovadoras, ativas e/ou novas formas de aprender e ensinar com 
o uso das tecnologias. Ao serem inseridos no mercado de trabalho, com raras exceções, a docência passa a se constituir por reprodução de práticas instauradas e cristalizadas no decorrer da formação discente e docente. Consideradas o "porto seguro" dos professores, as metodologias tradicionais, pautadas no professor "detentor do saber e reprodutor de conteúdos" e "alunos ouvintes", são como a bússola, instrumento de orientação que nunca apontará a direção incorretamente.

As formações continuadas tentam suprir esse importante papel de conscientizar/instrumentalizar o profissional docente para o potencial das tecnologias perante o público aprendente do século XXI, mas constituem-se como políticas de programas governamentais ou institucionais que atingem apenas uma parcela de um universo de profissionais a serviço da educação, no Brasil e no mundo. Por encontrar suas vozes mais fortes na formação continuada, o uso pedagógico de tecnologias na escola, é dotado de determinadas características que restringem seu poder de alcance e influência: o espaço-tempo destinado a ações formativas, o caráter não obrigatório de sua implementação, a falta de articulação das equipes gestoras e pedagógicas ante as políticas de uso de tecnologias educacionais e o apoio das instâncias governamentais na inclusão e fomento de uso de tecnologias e metodologias inovadoras nos documentos orientadores dos sistemas educacionais.

Tal paradigma é reforçado pela falta de uma linha teórica clara que subsidie o uso de tecnologias com a finalidade de promover o ensino e aprendizagem na escola e/ou a adoção de metodologias que transformem o aluno em autores e produtores de conhecimento. Dessa forma, o TPACK surge como uma base teórico-metodológica capaz de favorecer e ampliar as discussões sobre metodologias ativas, inovadoras e uso pedagógico de tecnologias.

\section{METODOLOGIA}

A metodologia desse estudo de natureza qualitativa, envolve a descrição sucinta de um processo formativo de professores do estado do Paraná, destacando as ações e observações de um grupo de cursistas participantes de oficinas sobre Aprendizagem Criativa e Introdução à Linguagem de Programação (Scratch). As formações constituíam-se por dois encontros presenciais de 4h cada, totalizando 8h de curso. De acordo com Marconi e Lakatos (2010), a abordagem qualitativa se constitui como uma pesquisa que analisa e interpreta informações, 
dados, com foco nos processos e seus significados. Dessa forma, esse tipo de pesquisa tem como objetivo entender os acontecimentos a partir da ótica do pesquisador.

As informações foram coletadas por meio de feedback oral dos professores ao término das formações e o principal questionamento das formadoras foi acerca da relação das atividades desenvolvidas durante as oficinas e o conteúdo disciplinar de cada participante.

\subsection{Formação continuada dos professores do estado do Paraná}

A Secretaria de Estado da Educação do Paraná (SEED), por meio da Coordenação de Tecnologia Educacional (CTE), lançou no ano de 2015/2016, o Projeto CONECTADOS que inicialmente, teve como objetivo principal a utilização pedagógica de tablets com alunos. $\mathrm{O}$ Núcleo Regional de Educação de Londrina participou do projeto piloto com 3 escolas localizadas no próprio município.

A escolha das escolas obedeceu critérios relacionados ao porte, localização e comprometimento dos gestores das respectivas instituições e contou com a adesão do estabelecimento de ensino, após consulta aos educadores e comunidade escolar. Ao aceitar os termos de adesão ao projeto, a instituição tinha como compromisso garantir a participação de professores e equipe pedagógica na formação continuada voltada ao uso pedagógico das tecnologias, bem como fomentar o desenvolvimento de ações com o uso de recursos tecnológicos em sala de aula.

A partir do ano de 2017, mais 24 escolas foram selecionadas para participar do projeto, que passou a se chamar CONECTADOS $2.0^{3}$, recebendo formação continuada voltada ao uso pedagógico das tecnologias educacionais e acompanhamento das ações desenvolvidas. $\mathrm{O}$ CONECTADOS 2.0 fez parte do programa de Formação Continuada da SEED e teve como objetivo principal favorecer as discussões sobre o uso de tecnologias educacionais além da busca pela implementação de uma cultura digital nas escolas que estabelecesse diálogos com metodologias ativas estimulando assim o protagonismo dos estudantes e um aprendizado baseado em experiências concretas.

O Projeto CONECTADOS 2.0 previu a distribuição de kits de equipamentos conforme o perfil de uso de cada escola, vinculando o recebimento destes à formação dos professores e à 
elaboração de um Plano de Ação Coletivo com Tecnologias Educacionais (PLACTEC). Para isso, direção, equipe pedagógica, alunos e professores responderam a algumas questões sobre o perfil da sua escola e, com isso, tiveram a autonomia de escolha dos seus equipamentos, para que, dessa maneira, a utilização fosse eficaz. Dentre as propostas de kits disponíveis, as escolas poderiam optar por Laboratórios de Produção (composto por câmera fotográfica com cartões de memória, tripé para câmera, caixa de som, fone de ouvido, gravador de áudio, notebook e impressora 3D), Rede sem fio, Laboratórios Móveis (netbooks conectados por meio de roteador móvel) e Projetores Multimídia. Ao fazer a escolha, se comprometeram a elaborar um plano de trabalho a partir do kit selecionado.

No ano de 2017, a sede do Departamento de Políticas e Tecnologias Educacionais (DPTE), da Secretaria de Estado da Educação do Paraná, em Curitiba, foi contemplado com um espaço "maker”, idealizado pelo então diretor de tecnologias educacionais do DPTE. O Seed Lab é um projeto selecionado pela Fundação Lemann e pelo Instituto de Tecnologia da Universidade de Massachusetts (MIT), por meio do Desafio Aprendizagem Criativa Brasil 2017. O Seed $\mathrm{Lab}^{4}$ oferece oficinas para professores e alunos, além de atender à comunidade com atividades de robótica, programação, construção de modelos, pequenos objetos, prototipagem para prova de conceito e simulações, produtos para as áreas do Design, da Engenharia e das Artes, mobiliário, máquinas e circuitos, desenvolvimento de produtos, projetos educacionais e compartilhamento do conhecimento.

Dando continuidade ao projeto CONECTADOS, o ano de 2018 foi dedicado a ações de formação dos professores da rede com ênfase em aprendizagem criativa, introdução à programação, segurança na internet e recursos educacionais Google, além de organização de eventos Scratch Day e participação dos formadores e professores da rede estadual em formações voltadas para a temática da aprendizagem criativa, conforme mostra de maneira sintetizada o quadro 1, a seguir, com as ações desenvolvidas pela SEED/DPTE e aplicadas pela CRTE (Coordenação Regional de Tecnologias na Educação) do Núcleo Regional de Educação de Londrina: 
Quadro 1 - Ações da SEED/DPTE - Tecnologias Educacionais (2016 a 2018)

\begin{tabular}{|c|c|c|}
\hline AÇ̃̃O & TEMAS & PARTICIPANTES \\
\hline $\begin{array}{c}14 \text { Oficinas }\left(1^{\circ} \text { semestre }\right) \\
5 \text { Oficinas }\left(2^{\circ} \text { semestre }\right) \\
\text { Email educacional } \\
\text { aescola:personalize sua } \\
\text { sala de aula }\end{array}$ & $\begin{array}{l}\text { Ferramentas do Google para a educação: } \\
\text { e-mail, drive, formulários e Google } \\
\text { Classroom (parceria com a Google para } \\
\text { a criação de contas educacionais). }\end{array}$ & $\begin{array}{l}\text { Professores, pedagogos e } \\
\text { agente educacional II } \\
\text { (servidor administrativo. }\end{array}$ \\
\hline $\begin{array}{l}5 \text { Oficinas }\left(2^{\circ} \text { semestre }\right) \\
\text { Descomplicando a } \\
\text { Programação }\end{array}$ & $\begin{array}{l}\text { Introdução lúdica à linguagem de } \\
\text { programação na escola com ênfase no } \\
\text { Scratch. }\end{array}$ & $\begin{array}{l}\text { Professores, pedagogos e } \\
\text { agente educacional II } \\
\text { (servidor administrativo). }\end{array}$ \\
\hline $\begin{array}{l}5 \text { Oficinas }\left(2^{\circ} \text { semestre }\right) \\
\text { Internet Segura }\end{array}$ & $\begin{array}{l}\text { Discussão sobre cyberbullying e crimes } \\
\text { virtuais e a importância de se tratar esta } \\
\text { temática na escola. }\end{array}$ & $\begin{array}{l}\text { Professores, pedagogos e } \\
\text { agente educacional II. }\end{array}$ \\
\hline $\begin{array}{l}4 \text { Oficinas }\left(2^{\circ} \text { semestre }\right) \\
\text { Estúdio de criação }\end{array}$ & $\begin{array}{l}\text { Formação voltada a estudar o conceito } \\
\text { de aprendizagem criativa e realizar } \\
\text { atividades "mão na massa" (movimento } \\
\text { maker) fazendo pontes para o currículo. }\end{array}$ & $\begin{array}{l}\text { Professores, pedagogos e } \\
\text { agente educacional II. }\end{array}$ \\
\hline $\begin{array}{l}24 \text { eventos } \\
\text { Scratch Day }\end{array}$ & $\begin{array}{l}\text { Dia dedicado ao desenvolvimento de } \\
\text { atividades com o Scratch para introduzir } \\
\text { e fomentar a programação na escola. }\end{array}$ & $\begin{array}{l}\text { Alunos do ensino regular } \\
\text { e das salas de recursos. }\end{array}$ \\
\hline $\begin{array}{l}\text { Formação: } \\
\text { Uso da impressora 3D }\end{array}$ & $\begin{array}{l}\text { Orientações técnicas quanto ao uso da } \\
\text { impressora 3D para as escolas que } \\
\text { receberam a impressora no kit do projeto } \\
\text { CONECTADOS 2.0. }\end{array}$ & $\begin{array}{l}\text { Professores e } \\
\text { funcionários dos } \\
\text { Colégios Estaduais José } \\
\text { C. Pinotti e Maria José B. } \\
\text { Aguilera. }\end{array}$ \\
\hline $\begin{array}{l}1^{\circ} \text { Conferência Brasileira } \\
\text { de Aprendizagem } \\
\text { Criativa }\end{array}$ & $\begin{array}{l}\text { Participação na } 1^{\circ} \text { Conferência } \\
\text { Brasileira de Aprendizagem Criativa } \\
\text { realizada em parceria com a Secretaria } \\
\text { de Estado da Educação (SEED). }\end{array}$ & $\begin{array}{l}\text { Formadores das CRTE } \\
\text { (Coordenação Regional } \\
\text { de Tecnologia na } \\
\text { Educação) e mais } 12 \\
\text { professores da rede } \\
\text { estadual. }\end{array}$ \\
\hline $\begin{array}{l}\text { Formação: Aprendizagem } \\
\text { Criativa }\end{array}$ & $\begin{array}{l}\text { Estudo teórico e prático aprendizagem } \\
\text { criativa em atividades "mão na massa". }\end{array}$ & $\begin{array}{l}3 \text { professores da rede } \\
\text { indicados pelos } \\
\text { formadores, para atuarem } \\
\text { como multiplicadores em } \\
\text { suas escolas. }\end{array}$ \\
\hline
\end{tabular}

Fonte: Acervo da pesquisa.

De acordo com os roteiros propostos pela Coordenação de Tecnologias Educacionais (CTE), a oficina Estúdio de Criação (Aprendizagem Criativa), implementada pela primeira 
autora deste artigo juntamente com a equipe de assessores pedagógicos, iniciou-se com a apresentação da base teórica em questão, empreendendo algumas reflexões com os professores sobre o tema e já na primeira etapa da oficina, composta por dois momentos de $4 \mathrm{~h}$, foram realizadas atividades "mão na massa" com os cursistas.

\section{RESULTADOS E DISCUSSÕES}

De modo geral, é possível constatar que o período de 2016 a 2018 foi muito produtivo para a área de tecnologias educacionais no estado do Paraná, uma vez que a Departamento de Políticas e Tecnologias Educacionais (DPTE) fomentou diversas ações com foco na Aprendizagem Criativa, movimento "maker" (mão na massa), iniciação à programação e ferramentas educacionais Google.

Em relação às oficinas ofertadas envolvendo as ferramentas do Google para a educação, tais como, e-mail, drive, formulários e Google Classroom, ficou evidente pela postura e falas dos professores participantes o engajamento e o interesse em descobrirem novas possibilidades tecnológicas que podem proporcionar atividades mais dinâmicas e interativas, despertando assim, o interesse do aluno, uma vez que tais recursos fazem parte do cotidiano dos estudantes dessa geração.

Um fato que nos chamou a atenção ocorreu na oficina de introdução à programação, pois percebemos que há uma tendência, observada pelos feedbacks dos professores ao final do curso, em considerar as atividades de programação potencialmente motivadoras e/ou facilitadoras das disciplinas das áreas Exatas. Os professores conseguiram identificar proximidade entre os conceitos de programação e os de Matemática e Física, por outro lado, os professores das áreas Humanas e Biológicas relataram que não conseguiram perceber relação entre as atividades desenvolvidas na oficina com sua área de atuação ou estas requerem um grande percurso entre os conhecimentos de programação até que consigam explorar com aluno uma atividade interligada ao seu objeto de aprendizagem, diferentemente dos conteúdos de Matemática e Física que, desde a elaboração inicial da programação, há possibilidade de se estabelecer pontes com o conteúdo curricular, segundo eles.

Quanto à oficina Estúdio de Criação, focada na proposta da Aprendizagem Criativa, essa experiência causou estranheza para os professores participantes pelo fato de não 
utilizarmos computadores durante as atividades. Esse estranhamento provavelmente ocorreu porque os cursos desenvolvidos pelo DPTE geralmente utilizam os computadores. No entanto, a nossa intenção era trabalhar o conceito de aprendizagem criativa por meio de atividades "mão na massa" (movimento maker), fazendo pontes com os conteúdos curriculares. Essa reação dos participantes nos alertou para a necessidade de abordar de forma mais ampla nas oficinas, práticas reflexivas - hands-on e head-in - e discussões teóricas que pudessem subsidiar os professores a compreender que o conceito aprendizagem criativa vai além do uso de uma determinada tecnologia. Importante é o professor compreender que a criação de situações de aprendizagem criativa deve estar pautada nos princípios construcionistas de Papert e tendo como pilares, na prática, os 4Ps de Resnick.

Para a oficina Estúdio de Criação, os profissionais (coordenadores e formadores) da Coordenação Regional (CRTE), instigados por diversos estudos e pesquisas, adaptaram algumas atividades propostas nas formações para contemplar as necessidades e os interesses dos professores das diferentes áreas do conhecimento. Assim, as atividades foram recriadas conforme elencamos a seguir:

Propostas criadas pela Coordenação Estadual:

- $\quad$ Criação de um pião (competição de giro do pião por mais tempo);

- $\quad$ Circuito em papel (iluminação de desenhos com LEDS);

- $\quad$ Luz de LED (ligar um LED com interruptor à fonte de energia).

Propostas criadas pelas formadoras:

- Viajante do tempo (entrevista com personagem histórico sem identificação para que os demais adivinhassem quem era a personalidade);

- Contação de histórias (criação de um personagem de uma narrativa com materiais alternativos);

- Marketing pessoal (criação de anúncio publicitário sobre si mesmo);

- $\quad$ Eu, publicitário (criação de objeto inédito no mercado);

- Mão robótica (montagem de uma mão robótica com materiais recicláveis para demonstrar o funcionamento de músculos e tendões).

Assim, procuramos contemplar atividades voltadas às disciplinas das áreas Humanas e Biológicas com o propósito de demonstrar que a aprendizagem criativa não se restringe às 
Exatas, embora esta área seja privilegiada pelas inúmeras oportunidades de práticas "mão na massa" oferecidas pelos conteúdos curriculares das disciplinas que a compõem. Percebe-se que o impacto dessas ações de formação continuada se consolida no esboço que se pode traçar das fragilidades teórico-metodológicas dos professores da rede pública de ensino em relação à Aprendizagem Criativa e o currículo. No entanto, o desafio continua em incentivar o professor a reconstruir seu conhecimento na perspectiva do TPACK, de modo que possa integrar as tecnologias (digitais ou não) com os conteúdos curriculares, na perspectiva enfatizada por Almeida e Valente (2011), atendendo as características contextuais da prática educativa.

\section{CONSIDERAÇÕES FINAIS}

A Diretoria de Políticas e Tecnologias Educacionais do Paraná, por meio de sua coordenação estadual e regionais, implementaram diversas ações para disseminar a Aprendizagem Criativa e fomentar entre os professores da rede pública a adoção de práticas metodológicas que ofereçam ao aluno estímulos a buscar informação e construir conhecimento, tornando-o protagonista de seu aprendizado.

A formação continuada com ênfase em metodologias ativas e inovadoras, de modo geral, foi bem recebida pelos professores da rede estadual, comprometidos com a melhoria da educação básica. No entanto, percebe-se que existem dificuldades dos professores em estabelecer relações com os conteúdos curriculares. Esse processo, que não é simples, requer reconstrução de conhecimentos que possam nortear as mudanças de posturas que a aprendizagem criativa exige, tais como: professor na função de mediador, reorganização dos espaços escolares, concepção de um currículo não linear, apoio da equipe de gestão escolar, dentre outros aspectos de ordem estrutural que interferem na mudança de perspectiva de uma educação centrada na mera transmissão de informação para uma educação em que centro do processo educativo está na interação e na mediação, tendo o aluno como protagonista da sua aprendizagem.

E o nosso papel, enquanto formadora e pesquisadora, é buscar aprender, refletindo, compartilhando, depurando, reformulando ações formativas que possam lançar novas sementes para que Aprendizagem Criativa possa oportunizar aos alunos o desenvolvimento da autonomia para aprender a aprender numa sociedade em que se exige de cada um a solidariedade e 
compromisso com a educação das crianças e dos jovens que serão os futuros profissionais de uma sociedade tecnológica.

\section{REFERÊNCIAS}

ACKERMANN, Edith. Tools for constructive learning: rethinking interactivity.

Massachusetts Institute of Technology Media Laboratory. Epistemology and Learning Group. Epistemology \& Learning Memo No. 15, 1993. Disponível em:

https://web.media.mit.edu/ edith/publications/1993-tools\%20for.const.\%20E\&L.pdf. Acesso em: 02 mar. 2020.

ALMEIDA, Maria Elizabeth Bianconcini de; VALENTE, José Armando. Tecnologias e currículo: Trajetórias convergentes ou divergentes? São Paulo: Paulus, 2011.

KOEHLER, Matthew J.; MISHRA, Punya. What is technological pedagogical content knowledge? Contemporary Issues in Technology and Teacher Education, v. 9, n. 1, 2009. Disponível em: http://www.citejournal.org/vol9/iss1/general/article1.cfm. Acesso em: 01 jul. 2019.

MISHRA, Punya; KOEHLER, Matthew. Technological Pedagogical Content Knowledge: A framework for teacher knowledge. Teachers College Record, 108(6), 1017-1054, 2006.

PAPERT, Seymour. A máquina das crianças. Porto Alegre: Artes Médicas, 1994.

PAPERT, Seymour; HAREL, Idit. Situating constructionism. In: HAREL, I.; PAPERT, S. (Ed.). Constructionism. Norwood: Ablex Publishing Corporation, 1991. Disponível em: http://www.papert.org/articles/SituatingConstructionism.html. Acesso em: 10 jul. 2019.

RESNICK, Mitchel. Give P'sachance: Projects, Peers, Passion, Play. In: Proceedings of Constructionism and Creativity Conference, Vienna, Austria, 2014.

SCHELLER, Morgana; VIALI, Lori; LAHM, Regis Alexandre. A Aprendizagem no contexto das tecnologias: uma reflexão para os dias atuais. CINTED- Novas Tecnologias na Educação, [S. l.], dezembro 2014. Disponível em: https://seer.ufrgs.br/renote/article/view/53513 . Acesso em: 11 jul. 2019.

SHULMAN, Lee. S. Knowledge and teaching: Foundations of the new reform. Harvard Educational Review, 57(1), pp. 1-22, 1987.

VALENTE, José Armando. Por quê o computador na educação. In: VALENTE, José Armando (Org.). Computadores e conhecimento: repensando a educação. 2. ed. Campinas: Gráfica Unicamp, 1998. p. 29-53. 
VALENTE, José Armando. Integração do pensamento computacional no currículo da educação básica: diferentes estratégias usadas e questões de formação de professores e avaliação do aluno. Revista e-Curriculum, São Paulo, v. 14, n. 3, p. 864-897, jul./set.2016. Disponível em: http://revistas.pucsp.br/index.php/curriculum. Acesso em 28 mar. 2020.

\section{NOTAS}

${ }^{1}$ Mitchel Resnick é diretor do grupo Lifelong Kindergarten, do Laboratório de Mídia do Instituto de Tecnologia de Massachusetts (MIT). Ele trabalhou por muitos anos com Seymour Papert, matemático que desenvolveu a teoria educacional Construcionismo.

${ }^{2}$ Veja livros do NIED-UNCAMP. Disponível em: www.nied.unicamp.br/biblioteca/o-computador-na-sociedadedo-conhecimento/.

${ }^{3}$ CONECTADOS 2.0. Disponível em:

http://www.gestaoescolar.diaadia.pr.gov.br/modules/conteudo/conteudo.php?conteudo=1731

${ }^{4}$ Seed Lab. Disponível em:

http://www.educadores.diaadia.pr.gov.br/modules/conteudo/conteudo.php?conteudo=1526

Recebido em: 13/04/2020

Aprovado em: 01/10/2020 